\title{
Assessment of a low-cost protocol for an ab initio based prediction of the mixing enthalpy at elevated temperatures: The Fe-Mo system
}

\author{
Kurt Lejaeghere, ${ }^{1}$ Stefaan Cottenier, ${ }^{1, *}$ Serge Claessens, ${ }^{2}$ Michel Waroquier, ${ }^{1}$ and Veronique Van Speybroeck ${ }^{1, \dagger}$ \\ ${ }^{1}$ Center for Molecular Modeling and QCMM Alliance Ghent-Brussels, Ghent University, Technologiepark 903, BE-9052 Zwijnaarde, Belgium \\ ${ }^{2}$ OCAS, Pres. J. F. Kennedylaan 3, BE-9060 Zelzate, Belgium \\ (Received 22 September 2010; revised manuscript received 18 February 2011; published 3 May 2011)
}

\begin{abstract}
We demonstrate how a limited number of $a b$ initio calculations in combination with a simple Debye model can predict a concentration- and temperature-dependent mixing enthalpy for a binary system. Fe-Mo is taken as a test case, and our predictions are compared with phase diagram information and a recently measured heat of solution for Mo in Fe. Crystallographic and magnetic information is calculated for the $\lambda$ and $\mu$ intermetallic phases in the Fe-Mo phase diagram as well. The present methodology can be useful for making a quick survey of mixing enthalpies in a large set of binary systems, in particular in the dilute concentration ranges where tabulated data are often lacking and where CALPHAD-style modeling is less reliable.
\end{abstract}

DOI: 10.1103/PhysRevB.83.184201

PACS number(s): 64.75.Ef, 81.30.Bx, 64.70.pe, 61.66.Dk

\section{INTRODUCTION}

The use of $a b$ initio methods in materials research has been booming over the past decade. ${ }^{1-6}$ Lattice parameters, elastic constants, and the saturation magnetization are just a few of the quantities that can now be routinely predicted from the fundamental equations of quantum physics, without a need for tunable parameters or experimental input. One particular niche in this field deals with the $a b$ initio prediction of thermodynamic quantities: pressure-volume relation, formation enthalpy, free energy, etc. ${ }^{6-9}$ This is particularly useful as it builds a bridge between two worlds: the behavior of individual atoms-which is the area where quantum physics applies - and the macroscopic world that is described by thermodynamics-which is the playground of material scientists.

In this paper, we aim for an ab initio based prediction of the concentration- and temperature-dependent enthalpy of mixing of a binary metallic alloy, and this at an affordable computational cost. The question is inspired by industrial research: in the search for new bulk metallic glasses (BMG), an empirical rule of thumb to identify potentially interesting candidate systems is to look for a negative enthalpy of mixing between the principal components. ${ }^{10}$ How can one obtain this information for a large set of candidate systems (assuming that only for a few of them tabulated data are available)? Performing experiments is an option, but a time-consuming one. Thermodynamic modeling (CALPHAD-style) can be an efficient alternative, but suffers from two shortcomings: not always all required input parameters are available (especially for multicomponent alloys), and such macroscopic models are likely to fail in the range of small concentrations (1-5\%) which is relevant for BMG systems. Both shortcomings do not apply to $a b$ initio methods, however: they are parameter free, and it is easier to apply them to dilute systems than to high-concentration alloys. On the other hand, a drawback of $a b$ initio methods is their inherent limitation to zero kelvin, whereas the mixing enthalpy that one is looking for is typically determined around the melting temperature.

As a specific study object, we take the Fe-Mo binary system. This choice is motivated by several reasons. In the context of
BMGs, the Mo content is an important parameter in a family of Fe-based metallic glasses. ${ }^{11}$ Mo is a well-known additive to specialty steel grades as well. The heat of solution of Mo in Fe has recently been measured experimentally, ${ }^{12}$ and has been computed by means of the semiempirical embedded atom method. ${ }^{13,14}$ Furthermore, the binary Fe-Mo phase diagram is well studied, ${ }^{15-20}$ and keeps raising interest. ${ }^{19-23}$

In Sec. II the methods that are applied in this work are presented, and their accuracy is tested in several ways. Section III summarizes the key properties of the Fe-Mo system, and gives an overview of the recent experimental and theoretical work on this system. In Secs. IV and V, our results for the formation enthalpy, the heat of solution and the mixing enthalpy are presented, at $0 \mathrm{~K}$ and at higher temperatures. The implications of these results are discussed in Sec. VI.

\section{METHODS AND ACCURACY TESTING}

Our aim is to make $a b$ initio based predictions of the enthalpy of mixing $\Delta H_{\text {mix }}(x)$ for a $\mathrm{Fe}_{1-x} \mathrm{Mo}_{x}$ alloy with an underlying bcc lattice, and this for all concentrations $0 \leqslant x \leqslant 1$ and at all relevant temperatures. (There is a possibility for terminology confusion at this point. What we mean by enthalpy of mixing is defined in Eq. (1). A synonym for this quantity is "integral enthalpy/heat of solution," or shortly "enthalpy/heat of solution." This is different from the "differential enthalpy/heat of solution" which we define in Eq. (6), and which is often called "enthalpy/heat of solution" for short as well.) The mixing enthalpy is defined as the enthalpy difference (per atom) between a configurationally disordered solid solution $\mathrm{Fe}_{1-x} \mathrm{Mo}_{x}$ (no short-range order) and a weighted average of the elemental materials:

$$
\begin{aligned}
\Delta H_{\text {mix }}(x)= & H\left(\mathrm{Fe}_{1-\mathrm{x}} \mathrm{Mo}_{\mathrm{x}}\right)_{\mathrm{random}} \\
& -[(1-x) H(\mathrm{Fe})+x H(\mathrm{Mo})] .
\end{aligned}
$$

(In this paper, $H, H_{\text {mix }}, H_{\text {form }}$, and $H_{\text {ord }}$ are always expressed per atom: $H_{\text {mix }}\left(A_{n} B_{m}\right)$ is the mixing enthalpy of one unit cell of $A_{n} B_{m}$, divided by the number of atoms in that unit cell. $H_{\mathrm{emb}}$ and $H_{\text {sol }}$ are expressed per impurity atom.) A solid solution is called ideal if its mixing enthalpy is zero. The deviation from 
ideality is the excess enthalpy (of mixing), which is obviously equal to the enthalpy of mixing itself. Similarly, the enthalpy of formation of an ordered FeMo alloy is defined as the enthalpy difference per atom between the ordered material $\mathrm{Fe}_{1-x} \mathrm{Mo}_{x}$ and a weighted average of the elemental materials:

$$
\begin{aligned}
\Delta H_{\text {form }}(x)= & H\left(\mathrm{Fe}_{1-x} \mathrm{Mo}_{x}\right)_{\text {ordered }} \\
& -[(1-x) H(\mathrm{Fe})+x H(\mathrm{Mo})] .
\end{aligned}
$$

The difference between the enthalpy of mixing and the enthalpy of formation is called the enthalpy of ordering, and represents the heat released when a randomly disordered solid solution evolves toward an ordered alloy:

$$
\begin{aligned}
\Delta H_{\text {ord }}(x) & =\Delta H_{\text {form }}(x)-\Delta H_{\text {mix }}(x) \\
& =H\left(\mathrm{Fe}_{1-x} \mathrm{Mo}_{x}\right)_{\text {ordered }}-H\left(\mathrm{Fe}_{1-x} \mathrm{Mo}_{x}\right)_{\text {random }} .
\end{aligned}
$$

At $0 \mathrm{~K}$ and without external pressure $p$, the enthalpy reduces to the ground state internal energy $U\left(V_{0}\right)$ at the equilibrium volume $V_{0}$. The same holds true for the Gibbs free enthalpy. Therefore, determining (excess) (free) enthalpies of mixing at zero pressure and temperature boils down to determining the internal energy of pure elemental solids and their mixtures. The internal energy is a quantity that can be computed by $a b$ initio methods (see further in this section). Solid state $a b$ initio calculations require a crystalline unit cell as input, by which an infinite three-dimensional lattice is built. For reasons of computer time, the number of atoms in such a unit cell should not be more than a few hundred. This is too small a number to mimic a truly disordered solid solution, and therefore the (free) enthalpy for the random mixture in Eq. (1) cannot be obtained right away. A common work-around is to derive the properties of a disordered compound from suitably chosen partially ordered compounds: the method of special quasirandom structures (SQS)..$^{6,9,24-26}$ Based on mixing enthalpy results obtained for many systems by SQS and other methods, Sluiter and Kawazoe ${ }^{26}$ concluded that the following approximate expression for the mixing enthalpy holds in many cases:

$$
\begin{aligned}
\Delta H_{\text {mix }}(x) \approx & x^{2}(1-x)\left[-\left.\frac{\partial \Delta H_{\text {mix }}}{\partial x}\right|_{x=1}\right] \\
& +x(1-x)^{2}\left[\left.\frac{\partial \Delta H_{\text {mix }}}{\partial x}\right|_{x=0}\right]
\end{aligned}
$$

The two terms in square brackets are the dilute limit derivatives of the mixing enthalpy, also known as the (differential) enthalpy/heat of solution for infinite dilution ${ }^{26}$ or the dilute impurity energy per solute atom. ${ }^{9}$ These derivatives can be approximated by finite difference ratios for low concentrations of either $\mathrm{Fe}$ or Mo. In this low concentration regime, the distinction between ordered and disordered solid solutions vanishes and Eqs. (1) and (2) evolve to the same values. Indeed, for sufficiently low impurity concentrations, the distance between two impurity atoms becomes large enough to prevent impurity-impurity interaction. In such cases, it does not matter anymore whether the impurity sublattice is ordered or not. This opens the door to ab initio calculations.

So-called "supercells" representing an ordered sublattice of impurities can be routinely dealt with by $a b$ initio methods: a $m \times m \times m$ supercell of the host material unit cell is taken, and a sparse sublattice is chosen on which the host material atoms are replaced by impurity atoms. The number of atoms in this large cell is called $n$, and, in the case of Mo impurities, the impurity fraction is given by $x=1 / n$. This supercell is the unit cell of a hypothetical ordered crystal, in which the impurity atoms are far away from each other. In terms of a supercell, the dilute limit of the heat of solution for Mo in Fe is expressed as

$$
\begin{aligned}
& \Delta H_{\mathrm{sol}}^{\mathrm{MocFe}}=\left.\frac{\partial \Delta H_{\mathrm{mix}}}{\partial x}\right|_{x=0}=\lim _{x \rightarrow 0} \frac{\Delta H_{\mathrm{mix}}(x)}{x} \\
&=\lim _{n \rightarrow \infty} H_{\mathrm{emb}}^{\mathrm{MocFe}} \\
&=\lim _{n \rightarrow \infty}\left\{n H\left(\mathrm{Fe}_{n-1} \mathrm{Mo}\right)-[(n-1) H(\mathrm{Fe})+H(\mathrm{Mo})]\right\}
\end{aligned}
$$

(and vice versa for $\mathrm{Fe}$ in Mo, with the opposite sign). The term in curly brackets in Eq. (8) defines the embedding enthalpy per solute atom for Mo in Fe-Mo alloys: the heat released upon inserting a single substitutional Mo impurity into a pure $n$-atom Fe cell. The same quantity divided by the number of atoms $n$ in the unit cell defines the formation enthalpy per atom:

$$
\begin{aligned}
\Delta H_{\text {form }}\left(\mathrm{Fe}_{n-1} \mathrm{Mo}\right)= & H\left(\mathrm{Fe}_{n-1} \mathrm{Mo}\right) \\
& -\left[\frac{n-1}{n} H(\mathrm{Fe})+\frac{1}{n} H(\mathrm{Mo})\right]
\end{aligned}
$$

and is identical to Eq. (2), but now expressed in terms of a supercell and therefore accessible by $a b$ initio calculations.

$A b$ initio calculations for a systematic series of cubic Fe-Mo supercells were done within the framework of density functional theory, ${ }^{27-29}$ using the Perdew-Burke-Ernzerhof exchange-correlation functional. ${ }^{30}$ For solving the scalarrelativistic Kohn-Sham equations we have employed the augmented plane waves + local orbitals $(\mathrm{APW}+\mathrm{lo})$ method $^{29,31,32}$ as implemented in the WIEN2k package ${ }^{33}$ for periodic solids. In this method the wave functions are expanded into spherical harmonics inside nonoverlapping atomic spheres of radius $R_{\mathrm{MT}}$ and in plane waves in the remaining space of the unit cell ( $=$ the interstitial region). We took $R_{\mathrm{MT}}^{\mathrm{Fe}}=R_{\mathrm{MT}}^{\mathrm{Mo}}=$ 2.20 a.u. in all cases, except for $\lambda$-Fe ${ }_{2}$ Mo where 2.00 a.u. was taken in order to avoid overlapping spheres. The maximum $\ell$ for the expansion of the wave function into spherical harmonics inside the spheres was taken to be $\ell_{\max }=10$. The charge density was Fourier expanded up to $G_{\max }=12 \sqrt{\mathrm{Ry}}$. Local orbitals were added for the Fe- $3 s$ and Fe-3 $p$ and the Mo- $4 s$ and Mo- $4 p$ "semicore" states. The plane wave expansion of the wave function in the interstitial region was truncated at a very large value of $K_{\max }=8.5 / R_{\mathrm{MT}}^{\min }=3.86$ a.u. $^{-1}$, which leads to very accurate values for the calculated energies. A very dense mesh of $k$ points (corresponding to a $52 \times 52 \times 52$ mesh for a conventional bcc unit cell) was taken, again leading to very accurate energies. These unusually stringent values for the basis set size $\left(K_{\max }\right)$ and $k$ mesh were necessary in order to get numerically converged values for the formation enthalpies at a given concentration $x$. For the same reason, the enthalpy per atom $H(\mathrm{Fe})$ for pure $\mathrm{Fe}$ in Eq. (8) was not calculated in a primitive unit cell, but in a pure Fe supercell with $n$ atoms. Otherwise, multiplication by the large factor $(n-1)$ would 
blow up any small numerical deviation in the enthalpy of the small cell.

The step from $a b$ initio calculated quantities at $0 \mathrm{~K}$ to quantities at higher temperatures is made by thermodynamics. The Gibbs free enthalpy $G$ for a given Mo concentration $x$ depends on pressure $p$ and temperature $T$, and is defined as

$$
\begin{aligned}
G_{x}(p, T)= & p V+U-T S \\
\approx & p V(\bar{\alpha})+\left[U_{\mathrm{el}}^{T=0}(\bar{\alpha})+U_{\mathrm{el}}^{T>0}(\bar{\alpha}, T)\right. \\
& \left.+U_{\mathrm{vib}}^{T=0}(\bar{\alpha})+U_{\mathrm{vib}}^{T>0}(\bar{\alpha}, T)\right] \\
& -T\left[S_{\mathrm{el}}(\bar{\alpha}, T)+S_{\mathrm{vib}}(\bar{\alpha}, T)+S_{\mathrm{conf}}(x)\right] .
\end{aligned}
$$

The symbol $\bar{\alpha}$ represents the geometry of the crystal (shape and size of the unit cell, and positions and identity of the atoms inside the unit cell), and is uniquely determined by $p$, $T$ and $x$. For any individual contribution to the free enthalpy, it is indicated whether it depends directly on $p$ and/or $T$, or rather indirectly via $\bar{\alpha}$, or both. For our purposes, the external pressure $p$ is zero. The zero temperature electronic energy $U_{\mathrm{el}}^{T=0}$ for the geometry $\bar{\alpha}$ is provided in a straightforward way by an $a b$ initio calculation. The zero-point vibrational energy $U_{\text {vib }}^{T=0}$ and the temperature-dependent vibrational energy and entropy sum up to the vibrational Helmholtz free energy $F_{\text {vib }}=U_{\text {vib }}-T S_{\text {vib }}$. Within the quasiharmonic approximation - where the phonon density of states $g(\omega, \bar{\alpha})$ is determined at every relevant volume- $-F_{\text {vib }}(\bar{\alpha}, T)$ can be expressed as an integral over $g(\omega, \bar{\alpha}) .{ }^{34-37}$ Obtaining $F_{\text {vib }}$ thus boils down to calculating the phonon density of states. The latter can be done at various levels of approximation. An accurate but rather time-consuming way is to compute the entire phonon spectrum. A much faster alternative is to apply the semiempirical Debye model. ${ }^{34}$ We opt for the latter, as it is consistent with our goal to obtain a decent estimate for the mixing enthalpy at an affordable computational cost. The expression for the Helmholtz vibrational free energy within the Debye model is given in Refs. 34 and 36, and it is shown in Ref. 36 that this leads to accurate temperature-dependent thermodynamical quantities for fcc $\mathrm{Ni}$ and $\mathrm{Ni}_{3} \mathrm{Al}$. Expressions for the electronic excitation energy $U_{\mathrm{el}}^{T>0}$ and related electronic entropy $S_{\mathrm{el}}$ are given in Refs. 38 and 36 . They require calculating the electronic chemical potential and the $0 \mathrm{~K}$ electron density of states at several volumes. Even though it is due to an excitation, $U_{\mathrm{el}}^{T>0}$ does not need to be positive: depending on the shape of the density of states, the redistribution of electrons according to a Fermi-Dirac distribution for $T>0$ may result in a band energy that is lower than the band energy for $0 \mathrm{~K}$ (see, e.g., Table III). These electronic contributions have been shown in Ref. 36 to be important for fcc $\mathrm{Ni}$ and $\mathrm{Ni}_{3} \mathrm{Al}$, where they affect enthalpy, heat capacity and thermal expansion in the range of $10-30 \%$. We have implemented the equations from Ref. 36, verified that we could reproduce their results for fcc $\mathrm{Ni}$, and will use them in Sec. $\mathrm{V}$ to determine these electronic contributions in the Fe-Mo system.

Removing the entropy terms from Eq. (10) yields the enthalpy $H$ for a geometry $\bar{\alpha}$ at pressure $p$ and temperature $T$ :

$$
\begin{aligned}
H_{x}(p, T)= & G_{x}(p, T)+T S_{\mathrm{tot}}(\bar{\alpha}, T, x) \\
\approx & p V(\bar{\alpha})+\left[U_{\mathrm{el}}^{T=0}(\bar{\alpha})+U_{\mathrm{el}}^{T>0}(\bar{\alpha}, T)\right. \\
& \left.+U_{\mathrm{vib}}^{T=0}(\bar{\alpha})+U_{\mathrm{vib}}^{T>0}(\bar{\alpha}, T)\right] .
\end{aligned}
$$

The enthalpy is the thermodynamic state function that is most directly accessible from heat exchange experiments.

\section{PROPERTIES OF THE Fe-Mo SYSTEM}

The low-temperature $(<800 \mathrm{~K})$ part of the Fe-Mo phase diagram can be found at several places, with minor differences. ${ }^{16-20}$ It contains the two elemental phases (bcc-Fe and bcc-Mo) and two crystalline intermetallic phases: the $\lambda$-phase $\mathrm{Fe}_{2} \mathrm{Mo}$ (C14 Laves phase, prototype $\mathrm{MgZn}_{2}$ ) and the $\mu$-phase $\mathrm{Fe}_{7} \mathrm{Mo}_{6}\left(\mathrm{D}_{5}\right.$, prototype $\left.\mathrm{Fe}_{7} \mathrm{~W}_{6}\right)$. Fe hardly dissolves in pure Mo (coexistence range $x=0.989-1.000$ at $800{ }^{\circ} \mathrm{C}^{20}$ ). For Mo concentrations between $x=0.46$ and $x=1.00$, there is coexistence between bcc-Mo and $\mathrm{Fe}_{7} \mathrm{Mo}_{6}$. Around $x=0.46$, there is a small homogeneity range for pure $\mathrm{Fe}_{7} \mathrm{Mo}_{6}\left(x=0.413-0.452\right.$ at $\left.800{ }^{\circ} \mathrm{C}^{20}\right)$. Below $x=0.46$, $\mathrm{Fe}_{2} \mathrm{Mo}$ and $\mathrm{Fe}_{7} \mathrm{Mo}_{6}$ coexist. At $x=0.33$, there is a narrow range for pure $\mathrm{Fe}_{2} \mathrm{Mo}\left(x=0.325-0.346\right.$ at $\left.800{ }^{\circ} \mathrm{C}^{20}\right)$. Below $x=0.33$, bcc-Fe and $\mathrm{Fe}_{2} \mathrm{Mo}$ coexist. Near $x=0.00$, there is a temperature-dependent solubility range for $\mathrm{Mo}$ in $\mathrm{Fe}$, with a width of a few percent $\left(x=0.000-0.037\right.$ at $\left.800{ }^{\circ} \mathrm{C}^{20}\right)$, and tending to zero (= no solubility) when extrapolated to $0 \mathrm{~K}$.

Several studies about various aspects of the Fe-Mo system have been reported during the past few years. The dilute limit of the differential heat of solution [Eq. (6)] at the Fe-rich side was recently determined by Mössbauer spectroscopy in combination with the Hrynkiewicz-Królas method. Mo concentrations as low as $1 \%$ were examined in samples that were slowly cooled after annealing. As diffusion stops at about $700 \mathrm{~K}$, the Mössbauer data that are taken at room temperature probe the atomic configuration and the corresponding value for $\Delta H_{\mathrm{sol}}^{\mathrm{Mo} \subset \mathrm{Fe}}$ at $700 \mathrm{~K}$. A strongly negative $\Delta H_{\mathrm{sol}}^{\mathrm{Mo} \subset \mathrm{Fe}}$ is found (see Table I). Three different theoretical approaches contribute a complementary piece of information (Table I). A cellular automata calculation found a weakly negative $\Delta H_{\mathrm{sol}}^{\mathrm{Mo} \subset \mathrm{Fe}}$, while an embedded atom method (EAM) calculation found a weakly positive value. A recent improved EAM calculation found a larger positive value. Both EAM versions rely on experimental input parameters that were obtained (mostly) at room temperature. They can therefore be considered to yield a $300 \mathrm{~K}$ result. The overall conclusion is that theory predicts Mo not to dissolve in $\mathrm{Fe}$ at zero kelvin, which is consistent with the experimental phase diagram. An $a b$ initio study in combination with the cluster expansion method (CEM), and based on a limited set of four intermetallic phases, found a

TABLE I. Experimental and theoretical values for the heat of solution of $\mathrm{Mo}$ in $\mathrm{Fe}$ as found in the literature and compared with results from the present work.

\begin{tabular}{lrccc}
\hline $\begin{array}{l}\Delta H_{\text {sol }} \\
(\mathrm{eV} / \text { atom })\end{array}$ & $T(\mathrm{~K})$ & exp/theory & method & year/Ref. \\
\hline$-0.645(80)$ & 700 & $\begin{array}{c}\text { exp } \\
\text { theory }\end{array}$ & $\begin{array}{c}\text { Mössbauer } \\
\text { cellular automata }\end{array}$ & $2010^{12}$ \\
-0.09 & & $1993^{39}$ \\
+0.03 & 300 & theory & EAM & $1993^{13}$ \\
+0.31 & 300 & theory & EAM & $2010^{14}$ \\
+0.11 & 0 & theory & DFT & this work \\
-0.34 & 700 & theory & DFT+QHD & this work \\
\hline \hline
\end{tabular}



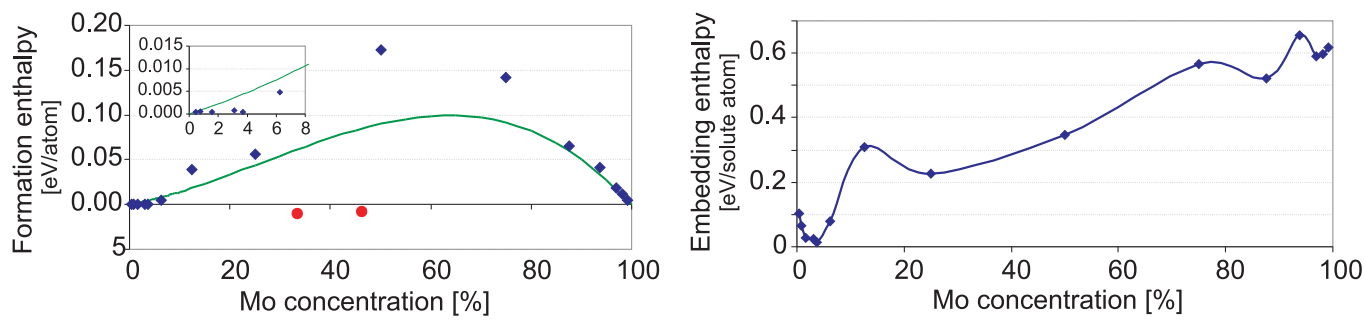

FIG. 1. (Color online) (a) Diamonds: Formation enthalpy at $0 \mathrm{~K}$ for cubic supercells as a function of Mo concentration. Circles: Formation enthalpy at $0 \mathrm{~K}$ for the $\lambda$ and $\mu$ intermetallic phases. Line: mixing enthalpy at $0 \mathrm{~K}$. Inset: detail of the concentration range $0-8 \%$. (b) Embedding enthalpy at $0 \mathrm{~K}$ as a function of Mo concentration.

miscibility gap extending over the entire concentration range at low temperature (Fig. 2-c in Ref. 23).

\section{RESULTS AT 0 K}

In a first series of calculations, no temperature effects were considered. The formation and embedding enthalpies were calculated as a function of concentration, using Eqs. (8) and (9) for a set of cubic supercells with either a simple cubic, bcc, or fcc sublattice of impurities. The largest supercell, corresponding to the lowest Mo concentration, contains 216 atoms $(x=0.0046)$. At the Mo-rich side, cells with up to 128 atoms $(x=0.9922)$ were considered. The results are shown in Fig. 1. The formation enthalpies for the cubic supercells are positive over the entire concentration range, indicating that $\mathrm{Fe}$ and Mo will never mix in an ordered cubic phase at $0 \mathrm{~K}$. This confirms what one expects from an extrapolation of the phase diagram to low temperatures. The formation enthalpies of the two known intermetallic phases are negative, indicating that $\lambda$ and $\mu$ are indeed stable ground state phases at the concentrations $x=0.333$ and $x=0.462$. The results of a full $a b$ initio structural optimization of the $\lambda$ and $\mu$ phases are given in Table II, and compared insofar as possible with experimental information. No entries for these two compounds are found at present in common crystallographic databases, ${ }^{40,41}$ such that the information on the internal positions that is given here might be their first explicit determination. Spin magnetic moments are reported as well in Table II: the $\lambda$ phase turns out to be an antiferromagnet, the $\mu$ phase is a ferromagnet. We are not aware of any experimental magnetometry studies on these compounds, and suggest this as a worthwhile task.

From the same set of data that provides the formation enthalpies in Fig. 1(a), embedding enthalpies are derived by means of Eqs. (7) and (8). They are shown in Fig. 1(b). There is an obvious difference in the convergence of the embedding enthalpies at the two edges of the concentration range. This is emphasized in Fig. 2, where the embedding enthalpy is plotted as a function of Mo-Mo or Fe-Fe distance, rather than as a function of Mo concentration. The embedding energy of $\mathrm{Fe}$ in Mo for a cell with 32 atoms is already near its value for infinite dilution (less than $0.03 \mathrm{eV}$ change per solute atom). For Mo in Fe, there is a further increase of $0.1 \mathrm{eV}$ per solute atom beyond a cell of 32 atoms, and even for a cell with 216 atoms convergence has not yet been fully reached. What is the reason for this, and why is it different at both extremes of the concentration range? A notable dependence of the embedding enthalpy on the concentration has been observed before. Wolverton and Ozolinšs ${ }^{9}$ examined the difference between embedding energies calculated for cells with 32 and 64 atoms, for 26 solute elements in fcc Al. Only in a small number of cases, differences of more than a few percent were observed. Erhart et al. ${ }^{42}$ examined the embedding enthalpy of $\mathrm{Cr}$ impurities in Fe for supercells up to 108 atoms ( $\mathrm{Cr}$ is isoelectronic with respect to $\mathrm{Mo})$. They considered not only cubic supercells, but more general types of supercells as well. For $\mathrm{Cr}$ concentrations below $x=0.03$,

TABLE II. Results of a full $a b$ initio structural optimization of the $\lambda$ (space group 194) and $\mu$ (space group 166, rhombohedral setting) intermetallic Fe-Mo phases. Experimental lattice parameters are taken from Ref. 20. Spin magnetic moments per atom are given within spheres with radius $R_{\mathrm{MT}}$. The total magnetic moment per formula unit (including the interstitial space) is $1.12 \mu_{B}$ for $\mathrm{Fe}_{2} \mathrm{Mo}$ and $8.52 \mu_{B}$ for $\mathrm{Fe}_{7} \mathrm{Mo}_{6}$.

\begin{tabular}{|c|c|c|c|c|c|c|c|c|}
\hline & $\mathrm{a}(\AA)$ & $\mathrm{c}(\AA)$ & Element & Site & $x$ & $y$ & $z$ & Moment $\left(\mu_{B}\right)$ \\
\hline $\mathrm{Fe}_{2} \mathrm{Mo}$ & 4.6594 & 7.7433 & $\mathrm{Fe}$ & $2 \mathrm{a}$ & 0.00000 & 0.00000 & 0.00000 & -1.13 \\
\hline \multirow[t]{2}{*}{$(\lambda)$} & & & $\mathrm{Fe}$ & $6 \mathrm{~h}$ & 0.17084 & 0.34169 & 0.75000 & +1.16 \\
\hline & & & Mo & $4 f$ & 0.33333 & 0.66667 & 0.06934 & -0.03 \\
\hline $\exp$ & $4.73(1)$ & $7.76(8)$ & & & & & & \\
\hline $\mathrm{Fe}_{7} \mathrm{Mo}_{6}$ & 9.0894 & & $\mathrm{Fe}$ & $1 \mathrm{a}$ & 0.00000 & 0.00000 & 0.00000 & +1.83 \\
\hline \multirow[t]{4}{*}{$(\mu)$} & $\alpha=30.23^{\circ}$ & & $\mathrm{Fe}$ & $6 \mathrm{~h}$ & 0.09063 & 0.58900 & 0.09063 & +1.27 \\
\hline & & & Mo & $2 \mathrm{c}$ & 0.16645 & 0.16645 & 0.16645 & -0.11 \\
\hline & & & Mo & $2 c$ & 0.34837 & 0.34837 & 0.34837 & +0.02 \\
\hline & & & Mo & $2 c$ & 0.45128 & 0.45128 & 0.45128 & -0.17 \\
\hline $\exp$ & $\begin{array}{c}9.013 \\
\alpha=30.64^{\circ}\end{array}$ & & & & & & & \\
\hline
\end{tabular}




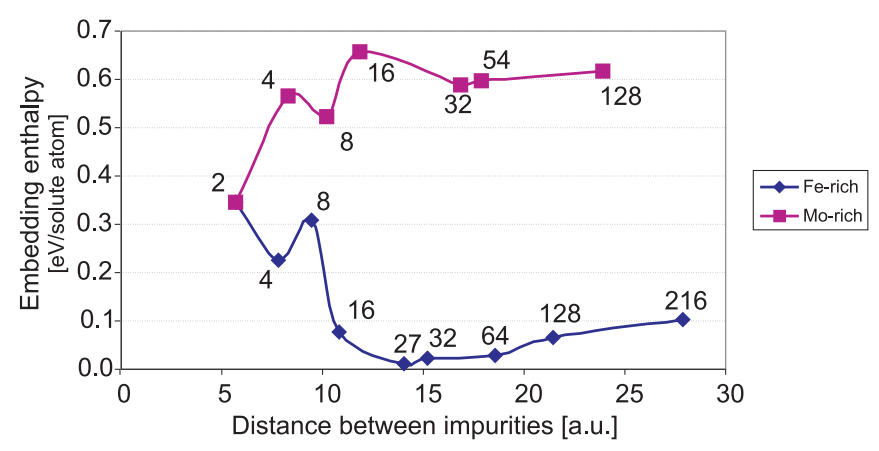

FIG. 2. (Color online) Embedding enthalpy at $0 \mathrm{~K}$ for Mo diluted in a Fe matrix (diamonds) and for Fe diluted in a Mo matrix (squares), plotted as a function of impurity-impurity distance. The labels at each point indicate the size of the supercell.

scatter in the embedding enthalpy was below $0.02 \mathrm{eV} /$ solute atom. The latter behavior is similar to what we observe at the Mo-rich side. The concentration dependence at the Fe-rich side, however, is significantly stronger. Wolverton and Ozolins $\check{s}^{9}$ attributed such concentration dependence to Friedel oscillations. We observe this mechanism to be at work in $\mathrm{Fe}-\mathrm{Mo}$ as well. A Mo impurity strongly perturbs the Fe spin moments, with an amplitude of $>0.1 \mu_{B}$ and a range that extends beyond the boundaries of the 216 atom cell. A Fe impurity in Mo, on the other hand, induces spin moments on the nonmagnetic Mo atoms in its neighbourhood that never exceed $0.03 \mu_{B}$ and rapidly fade out away from the Fe impurity. This means that an effective Mo-Mo interaction mediated by the region of strongly perturbed Fe moments acts over much longer distances than an effective $\mathrm{Fe}-\mathrm{Fe}$ interaction in a Mo matrix - which is what we observe as a slow saturation of the embedding enthalpy at the Fe-rich side.

\section{RESULTS AT HIGHER TEMPERATURES}

In a second series of calculations, the effect of temperature is taken into account by the procedure described in Sec. II. For two supercells ( $x=0.037$ and 0.969$)$ an accurate energy-vsvolume curve was calculated, using the same basis set size and $k$ mesh as for the determination of the embedding enthalpy, and optimizing the positions of the atoms inside the supercell at every volume. A Birch-Murnaghan equation of state was fit through these data, from which the equilibrium volume $V_{0}$, the static bulk modulus $B$ and its pressure derivative $B^{\prime}$ were obtained. These were used to determine the Debye temperature in the approximation of an isotropic liquid. ${ }^{43}$ This served as input for the quasiharmonic Debye model as implemented in the GIBBS code, ${ }^{43}$ to obtain the Gibbs free enthalpy [part of Eq. (10)] as a function of volume and temperature. At this stage, no electronic contributions were taken into account yet. At four temperatures- $0,500,1000$ and $1500 \mathrm{~K}$-the volume at which the minimum of the Gibbs free enthalpy is reached was determined. At these four volumes, the electronic density of states was $a b$ initio calculated, after which the electronic contributions in Eq. (10) were obtained (see Sec. II). By means of Eq. (11), the enthalpy of these systems at the four temperatures and corresponding volumes was found. The same procedure was applied to pure Fe and pure Mo as well. A selection of relevant quantities is listed in Table III.

$H_{\text {emb }}$ could now be obtained in the same way as at $0 \mathrm{~K}$ [Eqs. (7) and (8)]. The evolution of $H_{\mathrm{emb}}$ as a function of temperature is shown for $\mathrm{Fe}_{26} \mathrm{Mo}$ and $\mathrm{FeMo}_{31}$ in Fig. 3. The effect is significant for the Fe-rich alloy, and negligible for the Mo-rich alloy. Considering the fact that the Fe-rich and Morich compounds are close to pure Fe and pure Mo, respectively, we now make the approximation that the temperature induced shift for the dilute limits of the embedding enthalpy will be identical to the shift in these $x=0.037$ and $x=0.969$ alloys, respectively (one justification for this approximation is that relative trends tend to be more robust properties than absolute values). This finally gives us temperature-dependent solution enthalpies at both sides of the concentration range, from which we obtain by means of Eq. (5) a temperature-dependent expression for the mixing enthalpy. This is plotted for four relevant temperatures in Fig. 4.

In order to decide whether or not mixing will occur at a given concentration and temperature, the free enthalpy of mixing needs to be considered:

$$
\Delta G_{\mathrm{mix}}=\Delta H_{\mathrm{mix}}-T \Delta S_{\mathrm{mix}},
$$

$\Delta S_{\text {mix }}$ is the (configurational, vibrational, and electronic) entropy of the solid solution, minus the corresponding entropies of the pure phases. We have calculated the vibrational and electronic entropy for several ordered cubic $(\mathrm{Fe}, \mathrm{Mo})$ crystals, and found them to be at least one order of magnitude smaller than the ideal configurational entropy. Following, e.g., Ref. 44,

TABLE III. First four columns: equilibrium volume, bulk modulus, its pressure derivative, and electronic temperature-independent energy at equilibrium volume as result of a Birch-Murnaghan fit to calculated $E(V)$ data. $\theta_{D}$ : Debye temperature in the model of an isotropic elastic liquid. $V$ : unit cell volume at $T=500 \mathrm{~K}$ according to a purely vibrational quasiharmonic Debye model. $U_{e l}^{T>0}$ : temperature-dependent electronic contribution to the energy at $500 \mathrm{~K}$. $U_{\text {vib }}$ : vibrational contribution to the energy at $500 \mathrm{~K}$. The columns with energies serve as input in Eq. (11) to calculate enthalpies per atom.

\begin{tabular}{|c|c|c|c|c|c|c|c|c|}
\hline & \multicolumn{4}{|c|}{$0 \mathrm{~K}$} & \multirow[b]{2}{*}{$\begin{array}{c}\theta_{D} \\
(\mathrm{~K})\end{array}$} & \multicolumn{3}{|c|}{$500 \mathrm{~K}$} \\
\hline & $\begin{array}{c}V_{0} \\
\left(\AA^{3}\right)\end{array}$ & $\begin{array}{c}B \\
(\mathrm{GPa})\end{array}$ & $B^{\prime}$ & $\begin{array}{c}U_{e l}^{T=0}\left(V=V_{0}\right) \\
(\mathrm{eV} / \text { atom })\end{array}$ & & $\begin{array}{c}V \\
\left(\AA^{3}\right)\end{array}$ & $\begin{array}{c}U_{e l}^{T>0} \\
(\mathrm{eV} / \text { atom) }\end{array}$ & $\begin{array}{c}U_{\text {vib }} \\
(\mathrm{eV} / \text { atom) }\end{array}$ \\
\hline $\mathrm{Fe}$ & 11.372 & 200.0 & 6.0219 & -34634.7985 & 511.3 & 11.720 & 0.0065 & 0.0921 \\
\hline $\mathrm{Fe}_{26} \mathrm{Mo}$ & 313.809 & 198.4 & 4.2557 & -37433.3157 & 504.6 & 319.889 & -0.0007 & 0.0893 \\
\hline $\mathrm{FeMo}_{31}$ & 502.449 & 255.7 & 4.1836 & -107833.6754 & 443.4 & 507.771 & 0.0033 & 0.0926 \\
\hline Mo & 15.804 & 260.6 & 4.2217 & -110194.9485 & 445.1 & 15.968 & 0.0044 & 0.0925 \\
\hline
\end{tabular}



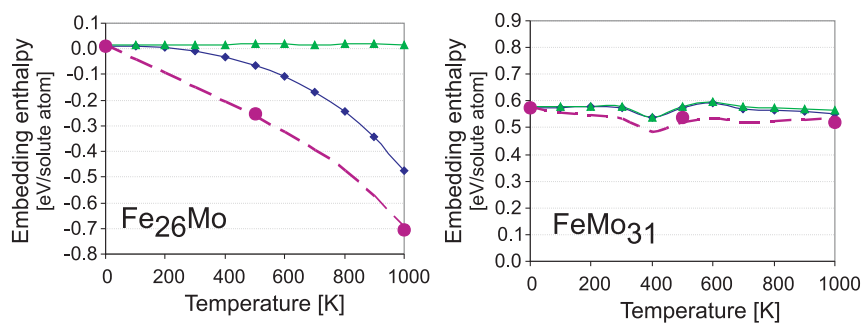

FIG. 3. (Color online) Embedding enthalpy as a function of temperature for representative Fe-rich and Mo-rich alloys. Diamonds: Debye model without electronic contribution. Circles: with electronic contribution. Triangles: without electronic contribution and in the approximation that the equation of state parameters for the alloy are a simple interpolation of the corresponding parameters for pure $\mathrm{Fe}$ and pure Mo.

we consider therefore the latter only. In this way, the free enthalpy of mixing is written as

$$
\begin{aligned}
\Delta G_{\text {mix }}= & \Delta H_{\operatorname{mix}} \\
& +k_{B} T[x \ln x+(1-x) \ln (1-x)] .
\end{aligned}
$$

This leads to a picture that is qualitatively similar to Fig. 4, but with all intersection points with the horizontal axis shifted by $10 \%(300 \mathrm{~K}), 15 \%(700 \mathrm{~K})$, or $20 \%(1000 \mathrm{~K})$ more to the right. The concentration intervals where mixing in the cubic phase is predicted to be favorable (i.e., negative $\Delta G_{\text {mix }}$ ) are summarized in Table IV. These predictions are consistent with the experimental observation that it is much easier to dissolve Mo in bcc-Fe than Fe in bcc-Mo. The results for intermediate concentrations cannot be directly compared to experiment due to the existence of the $\lambda$ and $\mu$ phases.

\section{DISCUSSION AND CONCLUSIONS}

How sophisticated a procedure does one need to apply in order to obtain at a moderate computational cost a fair $a b$ initio based estimate of the concentration- and temperaturedependent mixing enthalpy of a prototype binary alloy (FeMo)? For a $0 \mathrm{~K}$ assessment, a low-order polynomial ${ }^{26}$ based on solution enthalpies that are $a b$ initio calculated with supercells gives a result that is consistent with the available phase diagram

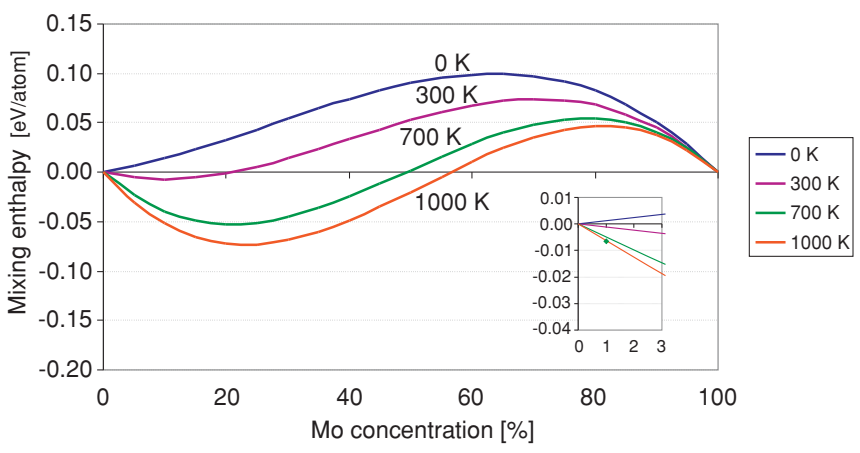

FIG. 4. (Color online) Mixing enthalpy at 0,300, 700, and $1000 \mathrm{~K}$ as a function of Mo concentration. The single point in the inset is the experimental value by Chojcan et al. at $1 \%$ and $700 \mathrm{~K},{ }^{12}$ obtained using their embedding enthalpy as the slope of a linear mixing enthalpy.
TABLE IV. Concentrations intervals where the free enthalpy of mixing [Eq. (13)] is found to be negative.

\begin{tabular}{rc}
\hline \hline$T(\mathrm{~K})$ & $x$ range \\
\hline 0 & - \\
300 & $0.00-0.30$ \\
700 & $0.00-0.65$ \\
1000 & $0.00-0.77$ \\
\hline
\end{tabular}

information: no mixing at all (Table I and Fig. 1(a)). Supercells with about 64 atoms provide reasonably accurate solution enthalpies, and by inspection of the perturbation of magnetic moments (if any) in the neighbourhood of the solute atom, one can assess whether this supercell size is sufficient or not. Numerically accurate values are obtained only when a large basis set and a dense Brillouin zone sampling are used. For the same reason of numerical accuracy, reference energies for the pure solvent matrix need to be calculated in supercells of the same size as for the solution enthalpy calculation. The advantage of the present method over the SQS method is that only two single-impurity supercell calculations are sufficient to provide the required input data for $0 \mathrm{~K}$ mixing enthalpies, and that no additional formalism is needed.

When temperature-dependent information is needed, a quasiharmonic Debye model with the inclusion of electronic correction terms $\mathrm{s}^{36}$ and configurational entropy [Eq. (13)] gives a result that is in qualitative agreement with experimental data (Table IV and Fig. 4). At room temperature, mixing occurs in the concentration range $0-30 \%$, with a minimal mixing enthalpy value of $-0.007 \mathrm{eV} /$ atom at $10 \% \mathrm{Mo}$ and a minimal value of $-0.016 \mathrm{eV} /$ atom for the free enthalpy of mixing at $15 \%$ Mo. If a more accurate prediction is needed, then a quasiharmonic model with ab initio calculated phonon spectra is the way to go. The computational cost is considerably larger though. Figure 3 and Table III demonstrate that without taking electronic terms into account it is impossible to obtain acceptable values for embedding and mixing enthalpies. Figure 3 demonstrates as well that a thermodynamic treatment of alloys based on structural parameters that are interpolations of the pure compounds leads to unacceptable results, even for small impurity concentrations.

We predict the $\lambda$ and $\mu$ phases to be stable ground state phases in the Fe-Mo phase diagram. A full prediction of their crystallographic properties is provided (Table II). $\lambda-\mathrm{Fe}_{2} \mathrm{Mo}$ is predicted to be an antiferromagnet, while $\mu-\mathrm{Fe}_{7} \mathrm{Mo}_{6}$ is a ferromagnet.

The procedure that is described here can be applied routinely to determine concentration- and temperaturedependent enthalpies of mixing in binary systems for which this information is not tabulated. The computational cost is moderate. Based on the present analysis for $\mathrm{Fe}$ Mo, we expect the resulting mixing enthalpy to be at least qualitatively in agreement with experiment, especially in the dilute ranges where CALPHAD-style modeling has limited predictive power. This procedure will be useful for situations where a larger set of candidate systems has to be scanned for promising properties (i.e., negative mixing enthalpy), as happens for instance in the search for new bulk metallic glasses. 


\section{ACKNOWLEDGMENTS}

This work is supported by the Fund for Scientific Research - Flanders (FWO) and by the Research Board of Ghent University. Computer resources and services were provided by Ghent University. *stefaan.cottenier@ugent.be

†veronique.vanspeybroeck@ugent.be

${ }^{1}$ J. Hafner, Acta Mater. 48, 71 (2000).

${ }^{2}$ M. Segall, P. Lindan, M. Probert, C. Pickard, P. Hasnip, S. Clark, and M. Payne, J. Phys. Condens. Matter 14, 2717 (2002).

${ }^{3}$ W. Liu, E. Karpov, S. Zhang, and H. Park, Comput. Methods Appl. Mech. Eng. 193, 1529 (2004).

${ }^{4}$ J. Hafner, C. Wolverton, and G. Ceder, MRS Bull. 31, 659 (2006).

${ }^{5}$ J. Hafner, J. Comput. Chem. 29, 2044 (2008).

${ }^{6}$ Z.-K. Liu, Journal Of Phase Equilibria And Diffusion 30, 517 (2009).

${ }^{7}$ L. S. Dubrovinsky, N. A. Dubrovinskaia, V. Swamy, J. Muscat, N. M. Harrison, R. Ahuja, B. Holm, and B. Johansson, Nature (London) 410, 653 (2001).

${ }^{8}$ K. Reuter and M. Scheffler, Phys. Rev. B 65, 035406 (2001).

${ }^{9}$ C. Wolverton and V. Ozoliņš, Phys. Rev. B 73, 144104 (2006).

${ }^{10}$ A. Inoue, Acta Mater. 48, 279 (2000).

${ }^{11}$ F. Liu, Q. Yang, S. Pang, and T. Zhang, J. Non-Cryst. Solids 355, 1444 (2009).

${ }^{12}$ J. Chojcan, R. Konieczny, A. Ostrasz, and R. Idczak, Hyperfine Interact. 196, 377 (2010).

${ }^{13}$ Z. Bangwei and O. Yifang, Phys. Rev. B 48, 3022 (1993).

${ }^{14}$ I. Dursun, Z. Güvenç, and E. Kasap, Commun. Nonlinear Sci. Numer. Simulat. 15, 1259 (2010).

${ }^{15}$ A. F. Guillermet, CALPHAD 6, 127 (1982).

${ }^{16} \mathrm{~J}$. Andersson, CALPHAD 12, 9 (1988).

${ }^{17}$ B. Predel, Landolt-Bornstein, Group IV Physical Chemistry-Phase Equilibria, Crystallographic and Thermodynamic Data of Binary Alloys, Vol. 5 (Springer-Verlag, Berlin, 1991-1998).

${ }^{18}$ Computational Thermodynamics, Iron-Molybdenum (Fe-Mo) Phase Diagram [http://www.calphad.com/iron-molybdenum .html].

${ }^{19}$ J. Houserová, J. Vrestál, and M. Sob, CALPHAD 29, 133 (2005).

${ }^{20}$ M. Eumann, G. Sauthoff, and M. Palm, Intermetallics 16, 706 (2008).

${ }^{21}$ A. Hirata and Y. Koyama, Phys. Rev. B 70, 134203 (2004).

${ }^{22}$ A. Hirata, A. Iwai, and Y. Koyama, Phys. Rev. B 74, 054204 (2006).

${ }^{23}$ N. Sodre, P. G. Gonzales-Ormeno, H. M. Petrilli, and C. G. Schoen, CALPHAD 33, 576 (2009).
${ }^{24}$ A. Zunger, S. H. Wei, L. G. Ferreira, and J. E. Bernard, Phys. Rev. Lett. 65, 353 (1990).

${ }^{25}$ S. H. Wei, L. G. Ferreira, J. E. Bernard, and A. Zunger, Phys. Rev. B 42, 9622 (1990).

${ }^{26}$ M. Sluiter and Y. Kawazoe, Europhys. Lett. 57, 526 (2002).

${ }^{27}$ P. Hohenberg and W. Kohn, Phys. Rev. 136, B864 (1964).

${ }^{28}$ W. Kohn and L. J. Sham, Phys. Rev. A 140, 1133 (1965).

${ }^{29}$ S. Cottenier, Density Functional Theory and the Family of $(L) A P W$ methods: a step-by-step introduction (Instituut voor Kern- en Stralingsfysica, KULeuven, Belgium, 2002) (freely available from [http://www.wien2k.at/reg_user/textbooks]).

${ }^{30}$ J. P. Perdew, K. Burke, and M. Ernzerhof, Phys. Rev. Lett. 77, 3865 (1996).

${ }^{31}$ E. Sjöstedt, L. Nordström, and D. J. Singh, Solid State Commun. 114, 15 (2000).

${ }^{32}$ G. K. H. Madsen, P. Blaha, K. Schwarz, E. Sjöstedt, and L. Nordström, Phys. Rev. B 64, 195134 (2001).

${ }^{33}$ P. Blaha, K. Schwarz, G. Madsen, D. Kvasnicka, and J. Luitz, WIEN2k, An Augmented Plane Wave + Local Orbitals Program for Calculating Crystal Properties (Karlheinz Schwarz, Techn. Universität Wien, Austria, 1999).

${ }^{34}$ A. van de Walle and G. Ceder, Rev. Mod. Phys. 74, 11 (2002).

${ }^{35}$ Y. Wang, Z. Liu, and L. Chen, Acta Mater. 52, 2665 (2004).

${ }^{36}$ S.-L. Shang, Y. Wang, D. Kim, and Z.-K. Liu, Comput. Mater. Sci. 47, 1040 (2010).

${ }^{37}$ S. Baroni, P. Giannozzi, and E. Isaev, Reviews in Mineralogy and Geochemistry 71, 39 (2010).

${ }^{38}$ C. Wolverton and A. Zunger, Phys. Rev. B 52, 8813 (1995).

${ }^{39}$ A. Miedema, Physica B: Condens. Matter 182, 1 (1993).

${ }^{40}$ ICSD (Inorganic Crystal Structure Database) (2010) [http://www. fiz-karlsruhe.de/icsd.html].

${ }^{41}$ COD (Crystallography Open Database) (2010) [http://www. crystallography.net/].

${ }^{42}$ P. Erhart, B. Sadigh, and A. Caro, Appl. Phys. Lett. 92, 141904 (2008).

${ }^{43}$ M. Blanco, E. Francisco, and V. Luaña, Comput. Phys. Commun. 158, 57 (2004).

${ }^{44}$ M. H. F. Sluiter, V. Vinograd, and Y. Kawazoe, Phys. Rev. B 70, 184120 (2004) 